TRANSACTIONS OF THE

AMERICAN MATHEMATICAL SOCIETY

Volume 348, Number 5, May 1996

\title{
GROUPS QUASI-ISOMETRIC TO COMPLEX HYPERBOLIC SPACE
}

\author{
RICHARD CHOW
}

\begin{abstract}
We show that any finitely generated group quasi-isometric to complex hyperbolic space is a finite extension of a properly discontinuous, cocompact subgroup of the isometry group.
\end{abstract}

\section{INTRODUCTION}

Two metric spaces are said to be quasi-isometric if there is a map from one space to the other whose image intersects every sufficiently large ball and which does not distort large-scale distances by more than a bounded factor. More precisely, metric spaces $\left(M, d_{M}\right)$ and $\left(N, d_{N}\right)$ are quasi-isometric if there are positive constants $C$, $K$, and $L$ and a function $\phi: M \rightarrow N$ satisfying:

(1) for all $x_{1}, x_{2} \in M$,

$$
\frac{1}{C} d_{M}\left(x_{1}, x_{2}\right)-K \leq d_{N}\left(\phi\left(x_{1}\right), \phi\left(x_{2}\right)\right) \leq C d_{M}\left(x_{1}, x_{2}\right)+K
$$

(2) for all $y \in N$,

$$
\left\{x: d_{N}(x, y) \leq L\right\} \cap \phi(M) \neq \emptyset .
$$

This definition is not given symmetrically, but it is not hard to see that if $M$ is quasi-isometric to $N$, then $N$ is quasi-isometric to $M$.

There is a natural metric on a finitely generated group, the word metric. The choice of a generating set for the group is necessary to define this metric, but different choices of generating sets will lead to quasi-isometric metrics.

Given a space, one would like to know which groups are quasi-isometric to the space. One class of examples is given by the observation that if a subgroup of the group of isometries acts properly discontinuously and cocompactly, then this subgroup is quasi-isometric to the space. One can simply fix a point $e$ in the space and define $\phi(g)=g(e)$ for an element $g$ of the subgroup. Of course, any finite extension of such a group will also be quasi-isometric to the space. Are these the only examples possible?

For $\mathbf{R}^{n}$ the answer is yes. By [Gro1], any group quasi-isometric to $\mathbf{R}^{n}$ is a finite extension of $\mathbf{Z}^{n}$. For real hyperbolic space $\mathbf{H}_{\mathbf{R}}^{n}$, the answer is also yes. For $n>2$, this follows from the work of Tukia [Tuk], Cannon and Cooper [C-C], and Gromov [Gro2]. The case of $n=2$ follows from the work of Gabai [Gab] or Casson and Jungreis $[\mathrm{C}-J]$ on convergence groups. Similarly, Pansu in [Pan] has shown that the

Received by the editors January 30, 1995 and, in revised form, May 4, 1995.

1991 Mathematics Subject Classification. Primary 20F32, 30C65.

Key words and phrases. Quasiconformal mappings, Heisenberg group, complex hyperbolic space, geometric group theory.

(C)1996 American Mathematical Society 
answer is also yes for the quaternionic and Cayley hyperbolic spaces. For further background, see [Ghy1], [Gro2], and [G-P]. Our aim here is to give a positive answer for the remaining rank one symmetric space, complex hyperbolic space $\mathbf{H}_{\mathrm{C}}^{n}$.

One point of view is that this question is about the existence side of Mostow rigidity. Mostow rigidity concerns the possibility of deforming a given lattice in a group. Here, we are given a lattice in rough form, as a group quasi-isometric to the associated symmetric space, and ask whether one can realize this rough lattice as a genuine lattice.

We note that for certain spaces a negative answer is known. That is, there are groups quasi-isometric to a space but which are not finite extensions of properly discontinuous, cocompact subgroups of the isometry group. An example is $\mathbf{H}_{\mathbf{R}}^{2} \times \mathbf{R}$; see [Rie] or [Ger].

The proofs for all the rank one symmetric spaces involve identifying a group quasi-isometric to the space with a group of "pseudo-isometries" acting on the space. This group of pseudo-isometries induces a group of quasiconformal maps on the boundary of the space. This quasiconformal group is then shown to be either conjugate via a quasiconformal mapping to a conformal group (in the real and complex hyperbolic cases) or a conformal group to begin with (in the quaternionic and Cayley cases). Since conformal mappings are the boundary values of isometries, this completes the proof.

The boundary of the complex hyperbolic spaces can be viewed as compactified Heisenberg groups. The main point here is that using results from Koranyi and Reimann's recent paper on quasiconformal mappings on the Heisenberg group [K-R1], we are able to show that Tukia's proof in the real hyperbolic case carries over to complex hyperbolic space.

We would like to thank Geoff Mess for suggesting this problem and for helpful conversations. We also are grateful to John Garnett and Eleanor Rieffel for encouragement and helpful conversations, and to the referee for corrections and suggestions.

\section{Preliminaries}

2.1. Complex Hyperbolic Space. One can concretely realize $n$-dimensional complex hyperbolic space $\mathbf{H}_{\mathbf{C}}^{n}$ as the unit ball in $\mathbf{C}^{n}$ with the Bergman metric. For a detailed description of the geometry, see [Gol] or [Eps]. The unitary group $S U(n, 1)$ acts holomorphically and isometrically on $\mathbf{H}_{\mathbf{C}}^{n}$. The action can be described as follows. Endow $\mathbf{C}^{n+1}$ with the quadratic form

$$
\langle z, z\rangle=-\left|z_{0}\right|^{2}+\sum_{j=1}^{n}\left|z_{j}\right|^{2} .
$$

Elements of $S U(n, 1)$ preserve this form and hence leave the cone

$$
\left\{z \in \mathbf{C}^{n+1}:\langle z, z\rangle<0\right\}
$$

invariant. Under projectivization, this cone can be identified with the unit ball. So if

$$
w_{j}=\frac{z_{j}}{z_{0}}, \quad j=1, \ldots, n,
$$

are nonhomogeneous coordinates, elements of $S U(n, 1)$ will induce a mapping of

$$
\left\{w \in \mathbf{C}^{n}:|w|<1\right\}
$$


to itself. As the isotropy subgroup of 0 is $U(n)$, one can also realize $\mathbf{H}_{\mathbf{C}}^{n}$ as the symmetric space $S U(n, 1) / U(n)$, where the natural metric agrees with the Bergman metric up to scale. If the metric is normalized so that the sectional curvature is "pinched" between -1 and $-1 / 4$, the planes corresponding to the exponential images of complex lines in the tangent space at any point are totally geodesic and have curvature -1 . They are isometric embeddings of $\mathbf{H}_{\mathbf{R}}^{2}$. The exponential images of planes in the totally real subspace have curvature $-1 / 4$.

The full isometry group of $\mathbf{H}_{\mathbf{C}}^{n}$ is generated by $S U(n, 1)$ and the anti-holomorphic mapping $\left(w_{1}, \ldots w_{n}\right) \rightarrow\left(\overline{w_{1}}, \ldots \overline{w_{n}}\right)$.

The metric on $\mathbf{H}_{\mathbf{C}}^{n}$ leads naturally to a "conformal" structure on its boundary $\partial \mathbf{H}_{\mathbf{C}}^{n}=S^{2 n-1}$ in much the same way as the real hyperbolic metric on the unit ball in $\mathbf{R}^{n}$ leads to the usual Euclidean conformal structure on its boundary, $S^{n-1}$. See $[\mathrm{G}-\mathrm{P}]$ for a sampling of various ways to derive this structure. Elements of the isometry group of $\mathbf{H}_{\mathbf{C}}^{n}$ extend to be conformal maps with respect to this structure, and conformal mappings all come from boundary mappings of isometries.

To describe concretely this conformal structure, we will work in coordinates. Via a generalized stereographic projection, $\partial \mathbf{H}_{\mathbf{C}}^{n} \backslash\{$ any point $\}$ can be identified with the Heisenberg group. One first maps the unit ball in $\mathbf{C}^{n}$

$$
\left\{\left(z_{1}, \ldots, z_{n-1}, \tau\right) \in \mathbf{C}^{n}:\left|\left(z_{1}, \ldots, z_{n-1}\right)\right|^{2}+|\tau|^{2}<1\right\}
$$

biholomorphically to the Siegel domain

$$
\left\{\left(z_{1}, \ldots, z_{n-1}, \tau\right) \in \mathbf{C}^{n}: \operatorname{Im} \tau>\left|\left(z_{1}, \ldots, z_{n-1}\right)\right|^{2}\right\} .
$$

Then $\partial \mathbf{H}_{\mathbf{C}}^{n} \backslash\{$ some point $\}$ will correspond to the boundary of the Siegel domain

$$
\left\{\operatorname{Im} \tau=\left|\left(z_{1}, \ldots, z_{n-1}\right)\right|^{2}\right\} .
$$

The boundary of the Siegel domain can then be mapped to $\mathbf{R}^{2 n-1}$ by sending

$$
\left(z_{1}, \ldots, z_{n-1}, \tau\right)=\left(x_{1}+i y_{1}, \ldots, x_{n-1}+i y_{n-1}, \tau\right)
$$

to

$$
\left(x_{1}, \ldots, x_{n-1}, y_{1}, \ldots, y_{n-1}, \operatorname{Re} \tau\right) .
$$

See [K-R2], section C, for the explicit formulae in the case of $\partial \mathbf{H}_{\mathbf{C}}^{2}$ where the point removed is $(0,1)$.

2.2. The Heisenberg Group. The Heisenberg group $H^{n}$ is a real $(2 n+1)$ dimensional Lie group. We model $H^{n}$ on $\mathbf{R}^{2 n+1}$ with coordinates $(x, y, t)$, where $x=\left(x_{1}, \ldots, x_{n}\right), y=\left(y_{1}, \ldots, y_{n}\right) \in \mathbf{R}^{n}$ and $t \in \mathbf{R}$. Alternatively, we can write $(z, t)$, where $z=\left(z_{1}, \ldots, z_{n}\right)=\left(x_{1}+i y_{1}, \ldots, x_{n}+i y_{n}\right)$. The group multiplication is given by

$$
(x, y, t)\left(x^{\prime}, y^{\prime}, t^{\prime}\right)=\left(x+x^{\prime}, y+y^{\prime}, t+t^{\prime}-2 x \cdot y^{\prime}+2 y \cdot x^{\prime}\right) .
$$

The invariant Haar measure on $H^{n}$ is the usual Lebesque measure $|\cdot|$ on $\mathbf{R}^{2 n+1}$. A basis for the Lie algebra of left-invariant vector fields is given by

$$
\begin{aligned}
X_{j} & =\frac{\partial}{\partial x_{j}}+2 y_{j} \frac{\partial}{\partial t}, \quad j=1, \ldots, n, \\
Y_{j} & =\frac{\partial}{\partial y_{j}}+2 x_{j} \frac{\partial}{\partial t}, \quad j=1, \ldots, n, \\
T & =\frac{\partial}{\partial t} .
\end{aligned}
$$


The only non-trivial commutator relations are $\left[X_{j}, Y_{j}\right]=-4 T, j=1, \ldots, n$.

A norm on $H^{n}$ is given by

$$
|(x, y, t)|=\left(\left(|x|^{2}+|y|^{2}\right)^{2}+t^{2}\right)^{1 / 4},
$$

thus giving the left-invariant distance function

$$
d(p, q)=\left|p^{-1} q\right| \text { for } p, q \in H^{n} .
$$

We use this distance function to define quasiconformality on $H^{n}$.

\subsection{Quasiconformal Maps on the Heisenberg Group.}

Definition. A homeomorphism $f: U \rightarrow V$ between domains in the Heisenberg group is quasiconformal if

$$
D(p)=\limsup _{r \rightarrow 0} \frac{\max _{d(p, q)=r} d(f(p), f(q))}{\min _{d(p, q)=r} d(f(p), f(q))}
$$

is uniformly bounded. Furthermore, $f$ is $K$-quasiconformal if $D(p) \leq K$ for almost every point $p \in H^{n}$.

A mapping of $\partial \mathbf{H}_{\mathbf{C}}^{n}$ to itself is $K$-quasiconformal if its restriction to any coordinate $H^{n-1}$ is $K$-quasiconformal. Conformal mappings by definition are 1quasiconformal mappings. Koranyi and Reimann [K-R2] have shown that isometries of $\mathbf{H}_{\mathbf{C}}^{n}$ act as conformal mappings on $\partial \mathbf{H}_{\mathbf{C}}^{n}$, and Pansu [Pan], Section 11.5, has shown that all conformal mappings on $\partial \mathbf{H}_{\mathbf{C}}^{n}$ are boundary values of isometries on $\mathbf{H}_{\mathrm{C}}^{n}$.

All the conformal mappings on the Heisenberg group are generated by the following groups. They all come from isometries of complex hyperbolic space.

(1) The Heisenberg group itelf: $H^{n}$ acts on itelf by left translation as a group of isometries and hence as a group of conformal mappings.

(2) A one-dimensional family of dilations around the origin:

$$
a_{s}:(x, y, t) \mapsto\left(e^{-s} x, e^{-s} y, e^{-2 s} t\right), \quad s \in \mathbf{R} .
$$

(3) Rotations around the origin:

$$
U(n) \in M:(z, t) \mapsto(M z, t) .
$$

(4) The inversion $(z, t) \mapsto(-\bar{z},-t)$, corresponding to an anti-holomorphic isometry of $\mathbf{H}_{\mathbf{C}}^{n}$.

The vector fields $\left\{X_{j}\right\}$ and $\left\{Y_{j}\right\}$ span a hyperplane, called the horizontal subspace, on the tangent space at each point of $H^{n}$ and provide $H^{n}$ with a contact structure. Conformal mappings are contact transformations with respect to this structure. See [K-R2].

2.4. Differentiability of quasiconformal mappings. Pansu has proven the fundamental theorem that quasiconformal mappings are differentiable almost everywhere, where the definition of differentiability is adapted to the group structure on the Heisenberg group. Recall that a map $f: R^{n} \rightarrow R^{n}$ which fixes the origin is differentiable at the origin if

$$
\lim _{a \rightarrow 0} \frac{f(a x)}{a}=M x
$$


for a linear transformation $M$. In the Heisenberg group setting, if $f$ fixes the origin, we say that $f$ is $\mathrm{P}$-differentiable at the origin in $H^{n}$ if the mappings

$$
a_{s}^{-1} \circ f \circ a_{s}
$$

converge locally uniformly as $s \rightarrow 0$ to a homomorphism of the Heisenberg group which preserves the horizontal space. P-differentiability at other points is defined via left translation. One can think of P-differentiability as meaning: (1) $f$ is differentiable in the Euclidean sense in horizontal directions and $D f$ takes the horizontal space to the horizontal space, and (2) $f$ is differentiable in the $T$ direction, where it behaves like $u \rightarrow \sqrt{u}$. See [G-P] and [K-R1].

The anisotropic nature of the metric on $\mathbf{H}_{\mathbf{C}}^{n}$ forces this "contact-map" nature of quasiconformal mappings. Indeed, even within the horizontal space, the derivative of a quasiconformal map is not arbitrary, owing to the difference in going in a complex line direction and going in a direction which is not a complex line. The derivative on the horizontal space must essentially be a symplectic matrix, those matrices preserving complex lines.

To be more precise, preserving the horizontal space forces the derivative $f_{*}$ to be a grade-preserving Lie algebra homomorphism. See [K-R1], Chapter 2. The grading of the Lie algebra $\mathbf{g}$ is given by

$$
\mathbf{g}=\mathbf{h} \oplus \mathbf{z},
$$

where $\mathbf{h}$ is the horizontal subspace and $\mathbf{z}$ is the span of $T$, the center of the Lie algebra. At almost every point this homomorphism is actually an automorphism. In the coordinates $\left\{X_{1}, \ldots, X_{n}, Y_{1}, \ldots, Y_{n}, T\right\}$ an orientation-preserving automorphism in terms of a matrix in $G L(2 n+1, \mathbf{R})$ can be given as

$$
\left(\begin{array}{cc}
\sqrt{\lambda} S & \\
& \lambda
\end{array}\right)
$$

where $\lambda>0$ and $S$ is an element of the symplectic group $S p(n, \mathbf{R})$, defined as those $2 n \times 2 n$ real matrices $A$ for which $A^{t} J A=J$ where $J=\left(\begin{array}{cc}I \\ -I\end{array}\right)$.

By definition, an orientation preserving automorphism is one which preserves the direction of $T$, i.e. $\lambda$ is positive. For orientation-reversing mappings, $\sqrt{\lambda}$ is replaced by

$$
\sqrt{-\lambda}\left(\begin{array}{cc}
-I & \\
& I
\end{array}\right)
$$

where I is the identity matrix on $\mathbf{R}^{n}$.

2.5. Conformal structures. Let $\mathcal{S}_{2 n}$ denote the symmetric space

$$
S L(2 n, \mathbf{R}) / S O(2 n) \text {. }
$$

One can identify $\mathcal{S}_{2 n}$ with the positive (symmetric, positive definite) $2 n \times 2 n$ real matrices of determinant one via the mapping $M \mapsto M^{t} M$. One can think of $\mathcal{S}_{2 n}$ as the space of ellipsoids in $2 n$-dimensional space or of positive-definite bilinear forms, both up to scale. $\mathcal{S}_{2 n}$ is a Riemannian manifold of nonpositive curvature on which $S L(2 n, \mathbf{R})$ acts isometrically by $X[A]=X^{t} A X$. The distance between the identity matrix $I$ and a positive matrix $A$ is given by

$$
K(A)=d(I, A)=\left(\left(\log \lambda_{1}\right)^{2}+\cdots+\left(\log \lambda_{n}\right)^{2}\right)^{1 / 2},
$$


where $\lambda_{1}, \ldots, \lambda_{n}$ are the eigenvalues of $A$. Other distances are given by the invariance under $S L(2 n, \mathbf{R})$.

The symmetric space $\mathcal{S}_{n}^{\star}=S p(n, \mathbf{R}) / S U(n)$ of positive symplectic matrices naturally sits inside $\mathcal{S}_{2 n}$ as a totally geodesic subspace. See [Ter] or [Maa].

We define a conformal structure $\mu$ on $U \subset \partial \mathbf{H}_{\mathbf{C}}^{n}$ to be an assignment to almost every $x \in U$ of an element of $\mathcal{S}_{n-1}^{\star}$ such that $K(\mu(x))$ is essentially bounded. A conformal structure is actually an almost everywhere defined field of bilinear forms on the horizontal subspace of the tangent space at each point. To identify an element of $\mathcal{S}_{n-1}^{\star}$ with a bilinear form, we need to work in particular Heisenberg coordinates, say on $\partial \mathbf{H}_{\mathbf{C}}^{n} \backslash(0, \ldots, 0,1)$. Hence, if $(0, \ldots, 0,1) \in U$, we prefer to let $\mu(0, \ldots, 0,1)$ remain undefined.

A quasiconformal mapping of $\partial \mathbf{H}_{\mathbf{C}}^{n}$ is conformal in $\mu$ if for a.e. $x \in \partial \mathbf{H}_{\mathbf{C}}^{n}$,

$$
\mu(x)=\left.f^{\prime}(x)\right|_{\mathbf{h}}[\mu(f(x))] .
$$

According to the previous section, the P-derivative of $f$ at $x$ is, in the orientationpreserving case, a positive multiple of a matrix in $S p(n, R)$ when restricted to the horizontal subspace. By an abuse of notation, we let $\left.f^{\prime}(x)\right|_{\mathbf{h}}$ denote this matrix. In the orientation-reversing case, the P-derivative of $f$ is a positive multiple of $\left(\begin{array}{cc}-I & \\ & I\end{array}\right) \cdot A$, where $A \in S p(n, \mathbf{R})$. The matrix $\left(\begin{array}{cc}-I & \\ & I\end{array}\right)$ corresponds to the mapping $\left(z_{1}, \ldots, z_{n}\right) \mapsto-\left(\overline{z_{1}}, \ldots, \overline{z_{n}}\right)$. In this case we once again normalize and ignore the positive factor.

We write $\mu_{g}(x)=\left.g^{\prime}(x)\right|_{\mathbf{h}}[\mu(x)]$. Hence $\mu_{f g}(x)=\left.g^{\prime}(x)\right|_{\mathbf{h}}\left[\mu_{f}(g(x))\right]$.

If $f: U \rightarrow \partial \mathbf{H}_{\mathbf{C}}^{n}$ is quasiconformal and $\mu$ is a conformal structure on $U$, then define the image $f_{*} \mu$ by

$$
f_{*} \mu(f(x))=\left(\left.f^{\prime}(x)\right|_{\mathbf{h}}\right)^{-1}[\mu(x)] .
$$

Note that

$$
(g f)_{*} \mu=g_{*} f_{*} \mu,
$$

when $f: U \rightarrow U^{\prime}$ and $g: U^{\prime} \rightarrow U^{\prime \prime}$ and $\mu$ is a conformal structure on $U$.

\section{Quasiconformal groups on the Heisenberg group}

A quasiconformal group of $\partial \mathbf{H}_{\mathbf{C}}^{n}$ is a group of quasiconformal mappings of $\partial \mathbf{H}_{\mathbf{C}}^{n}$ to itself such that every element is $K$-quasiconformal for some fixed $K$.

Tukia [Tuk] has shown that under certain conditions quasiconformal groups on $\overline{\mathbf{R}^{n}}$ are conjugate to a conformal group via a quasiconformal mapping. In this section our aim is to show that Tukia's theorems also hold in the Heisenberg group setting. The proofs of the theorems follow Tukia's general outline.

3.1. Lemmas on quasiconformal mappings. Let $f: D \rightarrow D^{\prime}$ be a homeomorphism of domains in $\mathbf{R}^{n}$, and for each open ball $B_{1} \subset D$ with center $x$, let $B_{2}$ denote the largest open ball in $f\left(B_{1}\right)$ with center $f(x)$. We say $f$ has the uniform density property if there exists a continuous $\phi:[0, \infty) \rightarrow[0, \infty)$ with $\phi(0)=0$ such that, for all $x \in D$ and sufficiently small $B_{1}$ with center at $x$,

$$
\frac{m\left(f(E) \cap B_{2}\right)}{m\left(B_{2}\right)} \leq \phi\left(\frac{m\left(E \cap B_{1}\right)}{m\left(B_{1}\right)}\right)
$$

for all measurable $E \subset D$. 
As in the Euclidean case, the uniform density property for quasiconformal mappings on the Heisenberg group is a consequence of a reverse Holder's inequality for the Jacobian. See $[\mathrm{G}-\mathrm{K}]$.

Lemma 3.1.1. Say $f: H^{n} \rightarrow H^{n}$ is K-quasiconformal. Then $f$ has the uniform density property with $\phi(t)=b t^{a}$, where $a$ and $b$ are positive constants, depending only on $K$ and $n$.

Proof. The proof is the same as in [G-K], since Koranyi and Reimann have proven that Gehring's $L^{p}$-integrability theorem for quasiconformal mappings on $\mathbf{R}^{n}$ also holds on $H^{n}$. For the reader's convenience, we reproduce the proof.

For $x \in H^{n}$, let $J_{f}(x)$ denote the absolute value of the Jacobian of $f$ at $x$, defined almost everywhere. By Theorem $\mathrm{G}$ in [K-R1], there exists a constant $C$, depending only on $K$ and $n$, such that

$$
\left(\frac{1}{|B|} \int_{B} J_{f}^{\frac{p}{2 n+2}} d v\right)^{\frac{2 n+2}{p}} \leq C \frac{1}{|B|} \int_{B} J_{f} d v
$$

for $p \in[2 n+2,2 n+2+\epsilon)$, some $\epsilon$, and for all balls $B$ in $H^{n}$.

Hence,

$$
\frac{1}{|B|} \int_{B} J_{f}^{\frac{p}{2 n+2}} d v \leq C^{\frac{p}{2 n+2}}\left(\frac{|f(B)|}{|B|}\right)^{\frac{p}{2 n+2}} .
$$

Fix $p=2 n+2+\epsilon / 2$. For $E$ measurable,

$$
\begin{aligned}
\left|f(E) \cap B_{2}\right| & \leq \int_{E \cap B_{1}} J_{f} d v \\
& \leq\left(\int_{B_{1}} J_{f}^{\frac{p}{2 n+2}} d v\right)^{\frac{2 n+2}{p}}\left|E \cap B_{1}\right|^{1-\frac{2 n+2}{p}} \\
& \leq C\left|B_{1}\right|^{\frac{2 n+2}{p}} \frac{\left|f\left(B_{1}\right)\right|}{\left|B_{1}\right|}\left|E \cap B_{1}\right|^{1-\frac{2 n+2}{p}} .
\end{aligned}
$$

Hence,

$$
\frac{\left|f(E) \cap B_{2}\right|}{\left|f\left(B_{1}\right)\right|} \leq C\left(\frac{\left|E \cap B_{1}\right|}{\left|B_{1}\right|}\right)^{1-\frac{2 n+2}{p}} .
$$

Since $\left|f\left(B_{1}\right)\right| \leq C^{\prime}\left|B_{2}\right|$ (see [K-R1], Proposition 12),

$$
\frac{\left|f(E) \cap B_{2}\right|}{\left|B_{2}\right|} \leq b\left(\frac{\left|E \cap B_{1}\right|}{\left|B_{1}\right|}\right)^{a} .
$$

The following two lemmas are due to Tukia in the $\mathbf{R}^{n}$ setting. His proofs go through with only minor modifications.

Lemma 3.1.2. Say $\mathcal{F}$ is a compact family of $K$-quasiconformal mappings from $\partial \mathbf{H}_{\mathbf{C}}^{n}$ to $\partial \mathbf{H}_{\mathbf{C}}^{n}$. Then there are positive constants $a, a^{\prime}, b$, and $b^{\prime}$, depending on $K$ and $\mathcal{F}$, such that

$$
b^{\prime} m(E)^{a^{\prime}} \leq m(f(E)) \leq b m(E)^{a}
$$

for all measurable $E$ and $f \in \mathcal{F}$. Here $m$ is the spherical measure.

Proof. It suffices to prove the right-hand inequality. We can assume the diameters of $E$ and $f(E)$ are bounded by 1 . Hence, we can assume $E$ and $f(E)$ are subsets of $H^{n-1}$ and use Euclidean measure $|\cdot|$, comparable to spherical measure on bounded sets. 
Let $r>0$. Let $r^{\prime \prime}=r^{\prime \prime}(x, r, f)>0$ be the biggest number such that $B\left(f(x), r^{\prime \prime}\right) \subset$ $f(B(x, r))$. Let $r^{\prime}=r^{\prime}(x, r, f)>0$ be the biggest number such that $f\left(B\left(x, r^{\prime}\right)\right) \subset$ $B\left(f(x), r^{\prime \prime}\right)$. Obviously, $r^{\prime}$ and $r^{\prime \prime}$ are continuous functions of $x, r$, and $f$. By the uniform density property, we can assume $r$ is so small that if $E \subset B\left(x, r^{\prime}\right)$ is measurable,

$$
\frac{|f(E)|}{\left|B\left(f(x), r^{\prime \prime}\right)\right|} \leq b\left(\frac{|E|}{|B(x, r)|}\right)^{a} .
$$

By compactness of $\mathcal{F}$ and because $x$ ranges over the compact set $\partial \mathbf{H}_{\mathbf{C}}^{n}, r^{\prime} \geq$ $r_{0}^{\prime}(\mathcal{F}) \geq 0$ and $r^{\prime \prime} \leq r_{0}^{\prime \prime}(\mathcal{F}) \leq \infty$.

So if $\operatorname{diam}(E)<r_{0}^{\prime}$,

for $f \in \mathcal{F}$.

$$
|f(E)| \leq \frac{\mid B\left(r_{0}^{\prime \prime} \mid\right.}{|B(r)|^{a}} b|E|^{a}
$$

Lemma 3.1.3. Say $\left\{f_{i}: \partial \mathbf{H}_{\mathbf{C}}^{n} \rightarrow \partial \mathbf{H}_{\mathbf{C}}^{n}\right\}$ is a family of K-quasiconformal mappings such that $f_{i}$ converges uniformly to $f: \partial \mathbf{H}_{\mathbf{C}}^{n} \rightarrow \partial \mathbf{H}_{\mathbf{C}}^{n}$. Say that for any $\epsilon>0$, $m\left(\left\{x: K\left(f_{i}(x)\right) \geq 1+\epsilon\right\}\right) \rightarrow 0$ as $i \rightarrow \infty$, where $m$ is the spherical measure. Then $f$ is conformal.

Proof. Note that Pansu's proof ([Pan], pp. 44-46), that 1-quasiconformal mappings on $\partial \mathbf{H}_{\mathbf{C}}^{n}$ come from isometries of $\mathbf{H}_{\mathbf{C}}^{n}$ relies only on the fact that 1-quasiconformal mappings preserve capacities. Hence, we need only show $f$ preserves capacities.

Recall that a ring in $\partial \mathbf{H}_{\mathbf{C}}^{n}$ is an open set with two complementary components. A ring on $H^{n}$ is, via stereographic projection, an open set with two complementary components, one of which contains $\infty$. The capacity of a ring $R$ with complementary components $C_{0}$ and $C_{1}$ is defined by:

$$
C(R)=\inf _{u} \int_{H^{n}}|\nabla u|^{2 n+2} d x,
$$

where $u$ ranges over all real-valued smooth functions on $H^{n}$ with $\left.u\right|_{C_{0}}=0$ and $\left.u\right|_{C_{1}}=1$, the so-called admissible functions. The expression

$$
\nabla u=\sum_{j=1}^{n}\left(\left(X_{j} u\right) X_{j}+\left(Y_{j} u\right) Y_{j}\right)
$$

is called the horizontal gradient of $u$, and

$$
|\nabla u|^{2}=\sum_{j=1}^{n}\left(\left|X_{j} u\right|^{2}+\left|Y_{j} u\right|^{2}\right)
$$

Let $A$ be a ring in $H^{n}$ with complementary components $C_{0}$ and $C_{1}$. Set, for $r>0$,

$$
A_{r}^{\prime}=\{z \in f(A): B(z, r) \subset f(A)\} .
$$

Then $f_{i}(A) \supset A_{r}^{\prime}$ for $i$ sufficiently large (depending on $r$ ).

Choose $\epsilon>0$. Let $u_{r}^{\prime}$ be admissible for $A_{r}^{\prime}$, so that

$$
\int_{A_{r}^{\prime}}\left|u_{r}^{\prime}\right|^{2 n+2} d m \leq C\left(A_{r}^{\prime}\right)+\epsilon .
$$

One can assume $u_{r}^{\prime}$ is smooth and is 0 on a neighborhood of $C_{0}$ and 1 on a neighborhood of $C_{1}$ by the proof of Proposition 11 in [K-R1]. Extend $u_{r}^{\prime}$ to $H^{n}$ by continuity and being constant on $H^{n} \backslash A_{r}^{\prime}$. 
Then clearly

for $A^{\prime} \supset A_{r}^{\prime}$.

$$
\int_{A_{r}^{\prime}}\left|\nabla u_{r}^{\prime}\right|^{2 n+2} d m=\int_{A^{\prime}}\left|\nabla u_{r}^{\prime}\right|^{2 n+2} d m
$$

Let $u_{i}=u_{r}^{\prime} \circ f_{i}$, admissible for $f_{i}^{-1}\left(A_{r}^{\prime}\right)$ and hence admissible for $A$.

Now, the inequality

$$
\left|\nabla u_{i}\right|^{2 n+2} \leq K^{2}\left(f_{i}, x\right) J_{f_{i}}\left|\nabla u_{r}^{\prime}(f(x))\right|^{2 n+2}
$$

follows from the chain rule. See [K-R1], p. 58 .

Set $E_{i}=\left\{x \in A: K^{2}\left(f_{i}, x\right) \geq 1+\epsilon\right\}$.

Then

$$
\begin{aligned}
C(A) & \leq \int_{A}\left|\nabla u_{r}^{\prime}\right|^{2 n+2} d m \\
& \leq \int_{A} K^{2}\left(f_{i}, x\right) J_{f_{i}}(x)\left|\nabla u_{r}^{\prime}(f(x))\right|^{2 n+2} d m \\
& \leq(1+\epsilon)^{2} \int_{f_{i}(A)}\left|\nabla u_{r}^{\prime}\right|^{2 n+2} d m+K^{2} \int_{f_{i}\left(E_{i}\right)}\left|\nabla u_{r}^{\prime}\right|^{2 n+2} d m .
\end{aligned}
$$

Since $\left|E_{i}\right| \rightarrow 0$ as $i \rightarrow \infty$, Lemma 3.1.2 implies $\left|f_{i}\left(E_{i}\right)\right| \rightarrow 0$ as $i \rightarrow \infty$. Hence the second term approaches 0 as $i \rightarrow \infty$.

Then,

$$
C(A) \leq(1+\epsilon)^{2}\left(C\left(A_{r}^{\prime}\right)+\epsilon\right) .
$$

So $C(A) \leq C\left(A_{r}^{\prime}\right)$ for small $r$. We claim that $C\left(A_{r}^{\prime}\right) \rightarrow C(f(A))$. Indeed, for any $\delta$ one can choose a smooth function $v$, compactly supported in $f(A)$, such that

$$
\int_{f(A)}|\nabla v|^{2 n+2} d m \leq C(f(A))+\delta
$$

But then, for small enough $r, v$ is also admissible for $C\left(A_{r}^{\prime}\right)$ and

$$
\int_{f(A)}|\nabla v|^{2 n+2} d m=\int_{A_{r}^{\prime}}|\nabla v|^{2 n+2} d m
$$

Since

$$
\int_{A_{r}^{\prime}}|\nabla v|^{2 n+2} d m \geq C\left(A_{r}^{\prime}\right) \geq C(f(A)) \geq \int_{f(A)}|\nabla v|^{2 n+2} d m+\delta
$$

and $\delta$ is arbitrary, $C(A) \leq C(f(A))$.

Since the assumptions of the lemma also hold for $f^{-1}, C(A)=C(f(A))$. Hence, capacities are preserved under $f$.

\subsection{Invariant conformal structures.}

Theorem 1. Let $G$ be a countable quasiconformal group on $\partial \mathbf{H}_{\mathbf{C}}^{n}$. Then there exists a conformal structure $\mu$ under which every element of $G$ is conformal.

Proof. $H^{n}$ has a measurable, $G$-invariant subset $U$ of full measure such that every $g \in G$ has non-zero P-derivative at all $x \in U$.

Set $M_{x}=\left\{\mu_{g}(x): g \in G\right\}$ for all $x \in U$. Then

$$
g^{\prime}\left[M_{g(x)}\right]=\left\{g^{\prime}(x)\left[\mu_{f}(g(x))\right]: f \in G\right\}=\left\{\mu_{g f}(x): f \in G\right\}=M_{x} .
$$

Recall Lemma E in [Tuk]: Let $N$ be a simply-connected, complete Riemannian manifold of nonpositive curvature. Suppose $X \subset N$ is non-empty and bounded. 
Then there is a unique disk with center $P_{X}$ which has the smallest radius of any disk containing $X$. Note that the phrase "negative curvature" in Tukia's paper really means nonpositive curvature.

We apply this lemma to $\mathcal{S}_{n}^{\star}$, which is complete and of negative curvature. Set $\mu(x)=P_{M_{x}}$. Since the $M_{x}$ as sets are invariant under $G$, the $P_{M_{x}}$ are also.

We need only show $\mu$ is measurable. Enumerate $G=\left\{g_{0}, g_{1}, \ldots\right\}$, and let $M(x, j)=\left\{\mu_{g_{i}}(x): i \leq j\right\}$ and $\mu_{j}(x)=P_{M(x, j)}$. Now, $X \mapsto P_{X}$ is continuous in the Hausdorff metric. This implies $\mu_{j}$ is measurable. Since $\mu_{j}(x)$ converges to $\mu(x), \mu$ is measurable.

3.3. Conjugating the group. Let $T=\left\{(u, v, w) \mid u, v, w \in \partial \mathbf{H}_{\mathbf{C}}^{n}\right.$, distinct $\}$. There is a natural projection $p$ from $T$ to $\mathbf{H}_{\mathbf{C}}^{n}$, namely the orthogonal projection of $w$ to the geodesic joining $u$ and $v$. We say $x$ is a radial point for the quasiconformal group $G$ if there are $g_{i} \in G$ such that for any $z \in T$ and geodesic $L \subset \mathbf{H}_{\mathbf{C}}^{n}$ with endpoint $x$,

in $\mathbf{H}_{\mathbf{C}}^{n}$ and

$$
z_{i}=p\left(g_{i}(z)\right) \rightarrow x
$$

$$
d\left(z_{i}, L\right) \leq M
$$

for some $M$.

A map $f: U \rightarrow X$, where $U \subset \partial \mathbf{H}_{\mathbf{C}}^{n}$ is open and $X$ is a metric space with metric $d$, is said to be approximately continuous at $x \in U$ if for all $\epsilon>0$

$$
m(\{y \in B(x, r) \cap U: d(f(x), f(y)) \leq \epsilon\}) / m(B(x, r)) \rightarrow 1
$$

as $r \rightarrow 0$. Here, $B(x, r)$ is the open ball in the spherical metric with radius $r$ and center $x$, and $m$ is the spherical measure. If $X$ is separable and $f$ is measurable, then $f$ is approximately continuous at almost every point. See [Fed].

Theorem 2. Let $G$ be a quasiconformal group of $\partial \mathbf{H}_{\mathbf{C}}^{n}$ and $\mu$ be a $G$-invariant conformal structure of $\partial \mathbf{H}_{\mathbf{C}}^{n}$. Say $\mu$ is approximately continuous at $x$, a radial point of $G$. Then there exists a quasiconformal mapping $f$ of $\partial \mathbf{H}_{\mathbf{C}}^{n}$ such that $f G f^{-1}$ is a conformal group of $\partial \mathbf{H}_{\mathbf{C}}^{n}$.

Proof. Let us take as our model for $\mathbf{H}_{\mathbf{C}}^{n}$ the unit ball in $\mathbf{C}^{n}$. The boundary $\partial \mathbf{H}_{\mathbf{C}}^{n}$ can then be identified with the unit sphere. Without loss, we assume $x=(1,0, \ldots, 0)$. Pick $g_{i} \in G$ and $z=\left(z_{1}, z_{2}, z_{3}\right) \in T$ which satisfy $(\star)$ for $M$ with respect to the line $L$ with endpoints $(1,0, \ldots, 0)$ and $(-1,0, \ldots, 0)$. Now we use Heisenberg coordinates $H^{n-1}$ on $\partial \mathbf{H}_{\mathbf{C}}^{n} \backslash(-1,0, \ldots, 0)$, for which $(1,0, \ldots, 0)$ will correspond to the identity $\mathbf{0}$ in the Heisenberg group.

Choose a smooth quasiconformal mapping $\alpha$ of $H^{n-1}$ fixing $\mathbf{0}$ such that $\alpha_{*} \mu(\mathbf{0})=$ I. Such an $\alpha$ exists because, for instance, one can take a linear map of the form (E1) or (E2) in Section 2.1.

Pick $s_{i}>0$ such that

$$
d\left((0, \ldots, 0), a_{s_{i}}\left(p g_{i}(z)\right)\right)=d\left((0, \ldots, 0), p\left(a_{s_{i}} g_{i}(z)\right)\right) \leq M,
$$

where $d(\cdot, \cdot)$ is the metric on $\mathbf{H}_{\mathbf{C}}^{n}$. Here, by an abuse of notation, $a_{s_{i}}$ means the isometry of $\mathbf{H}_{\mathbf{C}}^{n}$ which induces the $a_{s_{i}}$ defined in Section 2.3. Such $s_{i}$ exist since in $\mathbf{H}_{\mathbf{C}}^{n}$ the $a_{s_{i}}$ fix the geodesic $L$ and act as translations along $L$.

Define maps $f_{i}: \partial \mathbf{H}_{\mathbf{C}}^{n} \rightarrow \partial \mathbf{H}_{\mathbf{C}}^{n}$ by $f_{i}(u)=\alpha\left(a_{s_{i}}\left(g_{i}(u)\right)\right)$.

Along some subsequence $a_{s_{i}} g_{i} \rightarrow \bar{g}, \bar{g}$ quasiconformal, uniformly in the spherical metric. Indeed, first pick a subsequence so that $a_{s_{i}} g_{i}\left(z_{1}\right), a_{s_{i}} g_{i}\left(z_{2}\right)$, and $a_{s_{i}} g_{i}\left(z_{3}\right)$ 
converge. They must converge to distinct points, else (1) would be violated. Then apply [K-R1], p.67, Theorem F.

So $f_{i} \rightarrow f=\alpha \bar{g}$ uniformly in the spherical metric. We need only show $f g f^{-1}$ is conformal on $\partial \mathbf{H}_{\mathbf{C}}^{n}$ for all $g \in G$. Pick $g \in G$. Let $g^{\prime}=f g f^{-1}$ and

$$
g_{i}^{\prime}=f_{i} g f_{i}^{-1}=\left(a_{s_{i}} \alpha\right) g_{i} g g_{i}^{-1}\left(a_{s_{i}} \alpha\right)^{-1} .
$$

Consider the conformal structure $\mu_{i}=\left(f_{i}\right)_{*} \mu$ of $\partial \mathbf{H}_{\mathbf{C}}^{n}$. Each $g_{i}^{\prime}$ is conformal in this structure, so

$$
\mu_{i}(u)=\left(f_{i}\right)_{*} \mu(u)=\left(a_{s_{i}} \alpha\right)_{*}\left(g_{i}\right)_{*} \mu(u)=\left(a_{s_{i}} \alpha\right)_{*} \mu(u)=\alpha_{*} \mu\left(a_{s_{i}}^{-1}(u)\right),
$$

since $\left.a_{s_{i}}^{\prime}\right|_{\mathbf{h}}$ at any point is the identity matrix.

Since $\alpha_{*} \mu(\mathbf{0})=I$ and $\alpha_{*} \mu$ is approximately continuous at $\mathbf{0}$, given any $\epsilon>0$, there are sets $A_{i}$ such that $m\left(A_{i}\right) \rightarrow 0$ as $i \rightarrow \infty$ and $K\left(\mu_{i}(x)\right) \leq 1+\epsilon$ for $x \in \partial \mathbf{H}_{\mathbf{C}}^{n} \backslash A_{i}$.

The maps $g_{i}^{\prime}$ and $g^{\prime}$ are a compact family of quasiconformal mappings. Hence, by Lemma 3.1.2 there exists $B_{i} \subset \partial \mathbf{H}_{\mathbf{C}}^{n}$ such that $m\left(B_{i}\right) \rightarrow 0$ as $i \rightarrow \infty$ and $K\left(\mu_{i}(x)\right) \leq 1+\epsilon$ and $K\left(\mu_{i}\left(g_{i}^{\prime}(x)\right)\right) \leq 1+\epsilon$ for $x \in \partial \mathbf{H}_{\mathbf{C}}^{n} \backslash B_{i}$.

Since $g_{i}^{\prime}$ is conformal in $\mu_{i}$,

$$
\begin{aligned}
K\left(\left.g_{i}^{\prime}(x)\right|_{\mathbf{h}}\right) & \leq K\left(\mu_{i}(x)\right) K\left(\mu_{i}\left(g_{i}^{\prime}(x)\right)\right) \\
& \leq(1+\epsilon)^{2} \text { for } x \in \partial \mathbf{H}_{\mathbf{C}}^{n} \backslash B_{i} .
\end{aligned}
$$

Hence, $K\left(g_{i}^{\prime}(x)\right) \rightarrow 1$ in measure. Lemma 3.1.3 then implies $g^{\prime}=\lim _{i \rightarrow \infty} g_{i}^{\prime}$ is conformal.

\section{Main Theorem}

Theorem 3. A finitely generated group $G$ quasi-isometric to $\mathbf{H}_{\mathbf{C}}^{n}$ can be realized as the finite extension of a properly discontinuous, cocompact subgroup of the isometry group of $\mathbf{H}_{\mathbf{C}}^{n}$.

Proof. The hard work has all been done. Now we put together all the pieces. $G$ acts on itself freely by left translation as a group of isometries. Thus, each element of $G$ gives a quasi-isometry of $\mathbf{H}_{\mathbf{C}}^{n}$ to itself.

One of the steps in the proof of Mostow's basic theorem states that a quasiisometry between $\mathbf{H}_{\mathbf{C}}^{n}$ and itself induces a quasiconformal mapping of $\partial \mathbf{H}_{\mathbf{C}}^{n}$ to itself. See $[\mathrm{G}-\mathrm{P}]$ for a proof. The original proof due to Mostow [Mos] is in the case where the quasi-isometry is actually a bijective function. However, his proof easily generalizes to our situation. The main point is that an $M$-neighborhood of a geodesic must "map" to an $M^{\prime}$-neighborhood of another geodesic.

Hence, we have mapped $G$ to a group of quasiconformal mappings on $\partial \mathbf{H}_{\mathbf{C}}^{n}$. The kernel of this mapping is finite. To see this, take two geodesics in $\mathbf{H}_{\mathbf{C}}^{n}$ intersecting at the point $e$ corresponding to the identity of $G$. If $g \in G$ induces the identity on $\partial \mathbf{H}_{\mathbf{C}}^{n}$, these two geodesics will stay roughly fixed as sets, and hence $e$ cannot be moved very far by $g$ because it must stay near the intersection point of the two geodesics. But there are only a finite number of group elements mapped near $e$, and $G$ acts freely on itself.

Since we are only concerned with $G$ up to finite extension, we can identify $G$ with this quasiconformal group on $\partial \mathbf{H}_{\mathbf{C}}^{n}$.

Lemma 4.0.1. Every point of $\partial \mathbf{H}_{\mathbf{C}}^{n}$ is radial with respect to $G$. 
Proof of Lemma. Given any $x \in \partial \mathbf{H}_{\mathbf{C}}^{n}$ and any geodesic $L \subset \mathbf{H}_{\mathbf{C}}^{n}$ with endpoint $x$, we can find $\left\{g_{i}\right\} \in G$ such that $g_{i}(e)$ converges to $x$ and $d\left(g_{i}(e), L\right) \leq M$, where $e$ is the point in $\mathbf{H}_{\mathbf{C}}^{n}$ corresponding to the identity of $G$ and $M$ is a constant depending on the quasi-isometry constants. Take a triple $(u, v, w)=t \in T$ such that $p(t)=e$. Now there is a constant $C$ such that

$$
d\left(p g_{i}(t), g_{i} p(t)\right) \leq C \text {. }
$$

To see this, take an isometry $h$ of $\mathbf{H}_{\mathbf{C}}^{n}$ such that $h g_{i}(t)=t$. Then

$$
d\left(g_{i} p(t), p g_{i}(t)\right)=d\left(h g_{i} p(t), p(t)\right) .
$$

$h g_{i}$ is a quasi-isometry which fixes $u, v$, and $w$. Hence, it is easy to see it cannot move their projection very far. For a precise argument, see [Thu], Lemma 5.9.4. Then $p\left(g_{i}(t)\right)$ converges to $x$ and $d\left(p\left(g_{i}(e)\right), L\right) \leq M^{\prime}$.

Hence, Theorem 2 implies that $G$ is conjugate to a group of isometries of $\mathbf{H}_{\mathbf{C}}^{n}$ via a quasiconformal mapping $f$. We note that $f$ can be extended to a quasi-isometry

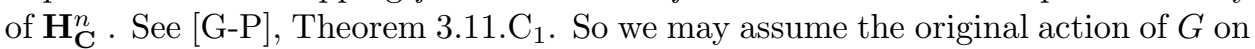
$\partial \mathbf{H}_{\mathbf{C}}^{n}$ is conformal, since if $\phi$ is the original quasi-isometry between $G$ and $\mathbf{H}_{\mathbf{C}}^{n}$, we may replace it by $f \circ \phi$. Hence, we have identified $G$ with a group of isometries of $\mathbf{H}_{\mathbf{C}}^{n}$; we need only show this group acts properly discontinuously and cocompactly. But this metric-space argument is contained in Cannon and Cooper's paper [C-C] as steps (4) and (5) in the proof of their main theorem. Paraphrasing them, the argument succeeds because the action of $G$ on itself is cocompact and properly discontinuous, and $\mathbf{H}_{\mathbf{C}}^{n}$ and $G$ are equivalent, up to the factors that matter in the definitions of proper discontinuity and cocompactness.

Richard Schwartz [Sch] has proven that any group quasi-isometric to a nonuniform rank one lattice is virtually a rank one lattice. Schwartz's work does not cover the case of $S L(2, \mathbf{R})$, but this case was known before, as here non-uniform lattices are virtually free groups. Any group quasi-isometric to a free group is virtually a free group; see for example [Ghy2]. He points out that since we have completed the classification of groups quasi-isometric to uniform rank one lattices, we have the following:

Corollary. Any finitely generated group quasi-isometric to a rank one lattice is virtually a rank one lattice.

\section{REFERENCES}

[C-C] J. W. Cannon and D. Cooper, A characterization of cocompact hyperbolic and finitevolume hyperbolic groups in dimension three, Trans. Amer. Math. Soc. 330 (1992), 419431. MR 92f: 22017

[C-J] A. Casson and D. Jungreis, Convergence groups and Seifert fibered 3-manifolds, Inventiones Math. 118 (1994), 441-456. CMP 95:01

[Eps] D. B. A. Epstein, Complex hyperbolic geometry, Analytical and Geometric Aspects of Hyperbolic Space, London Mathematical Society Lecture Notes, 111, Cambridge University Press, Cambridge (1987), 93-111. MR 89c:32082

[Fed] H. Federer, Geometric Measure Theory, Springer-Verlag, Berlin, 1969. MR 41:1976

[Gab] D. Gabai, Convergence groups are Fuchsian groups, Annals of Math. 136 (1992), 447510. MR 93m:20065

[G-K] F. W. Gehring and J. C. Kelly, Quasi-conformal mappings and Lebesgue density, Discontinuous Groups and Riemann Surfaces, Proceedings of the 1973 Conference at the University of Maryland (L. Greenberg, ed.), Ann. of Math. Studies 79, Princeton University Press (1974), 171-179. 
[Ger] S. M. Gersten, Bounded cohomology and combings of groups, Int. J. Algebra Comput. 2 (1992), 307-326. MR 93i:20029

[Ghy1] E. Ghys, Les groupes hyperboliques, Seminaire Bourbaki 722 (1990), 1-29. MR 92f:57004

[Ghy2] E. Ghys and P. de la Harpe (eds.), Sur les groupes hyperboliques d'après Mikhael Gromov, Progress in Mathematics 83, Birkhäuser (1990). MR 92f:53050

[Gol] W. M. Goldman, Complex Hyperbolic Geometry, preprint.

[Gro1] M. Gromov, Groups of polynomial growth and expanding maps, Publ. Math. IHES 53 (1981), 53-73. MR 83b:53041

[Gro2] Hyperbolic groups, Essays in Group Theory (S. M. Gersten, ed.), MSRI 8, Springer (1987), 75-263. MR 89e:20076

[G-P] M. Gromov and P. Pansu, Rigidity of lattices: an introduction, Geometric Topology: Recent Developments (J. Cheeger et. al., eds.), Lecture Notes in Math., vol. 1504, SpringerVerlag, Berlin and New York, 1991, pp. 39-137. MR 93f:53036

[K-R1] A. Koranyi and H. M. Reimann, Foundations for the theory of quasiconformal mappings on the Heisenberg group, Advances in Math. 111 (1995), 1-87.

[K-R2] Q Quasiconformal mappings on the Heisenberg group, Inventiones Math. 80 (1985), 309-338. MR 86m:32035

[Maa] H. Maass, Siegel's Modular Forms and Dirichlet Series, Lecture Notes in Mathematics 216, Springer-Verlag, Berlin, 1971. MR 49:8938

[Mos] G. D. Mostow, Quasi-conformal mappings in n-space and the rigidity of hyperbolic space forms, Publ. Math. IHES 34 (1968), 53-104. MR 38:4679

[Pan] P. Pansu, Metriques de Carnot-Caratheodory et quasi-isometries des espaces de rang 1, Annals of Math. 129 (1989), 1-60. MR 90e:53058

[Rie] E. Rieffel, Groups coarse quasi-isometric to $\mathbf{H}^{2} \times \mathbf{R}$, preprint.

[Sch] R. Schwartz, The quasi-isometry classification of rank one lattices, Publ. I.H.E.S. (to appear).

[Ter] A. Terras, Harmonic analysis on symmetric spaces II, Springer-Verlag, New York, 1988. MR 89k:22017

[Thu] W. P. Thurston, The geometry and topology of 3-manifolds, Lecture Notes, Princeton Univ., 1978.

[Tuk] P. Tukia, On quasiconformal groups, Journal D'Analyse Math. 46 (1986), 318-346. MR 87m:30043

Department of Mathematics, National University of Singapore, Singapore 0511

Current address: Department of Mathematics, University of California, Los Angeles, California 90024

E-mail address: rchow@math.ucla.edu 